\title{
By-emitter Emulation Enhancement Tool Using a Global Thermal Solver for the Degradation Emulation of a Calibrated 975 nm Tapered Laser Bar
}

\author{
Christian Kwaku Amuzuvi $^{1,2, *}$, Joseph Cudjoe Attachie ${ }^{1}$, Kofi Asante $^{1}$ \\ ${ }^{1}$ Department of Electrical and Electronic Engineering, University of Mines and Technology, Tarkwa, Ghana \\ ${ }^{2}$ Photonic and Radio Frequency Engineering Group (PRFEG), Electrical Systems and Optics Research Division, Faculty of \\ Engineering, University of Nottingham, Nottingham, United Kingdom \\ *Corresponding author: ckamuzuvi2000@yahoo.com
}

Received August 23, 2013; Revised November 15, 2013; Accepted November 21, 2013

\begin{abstract}
In this paper, Barlase has been taken a step further by emulating the degradation processes in high power semiconductor laser bars using an upgraded version of Barlase by the introduction of a global thermal solver to further deepen the understanding of the behaviour of laser bars. In this paper, the emulation of a real laser bar was investigated to emulate experimental results by simulating the experimental results in the view of finding a correlation between them. The results established show a more elaborate frown shaped power/current profile and a corresponding frown shaped temperature profile especially at the front facet of the laser bar. Even though a more elaborate frown shaped profile was realised in the power, current and temperature profiles, it fell short from what was seen in the experimental results. As the emulation of laser bar degradation has not been attempted before, further work is needed to achieve better agreement in the output power, current and temperature profiles to better the model.
\end{abstract}

Keywords: by-emitter, emitter, calibration, tapered laser bar, power, Heatsink temperature, electroluminescence, near infra-red, degradation, trap density

Cite This Article: Christian Kwaku Amuzuvi, Joseph Cudjoe Attachie, and Kofi Asante, "By-emitter Emulation Enhancement Tool Using a Global Thermal Solver for the Degradation Emulation of a Calibrated 975 nm Tapered Laser Bar.” American Journal of Electrical and Electronic Engineering 1, no. 3 (2013): 60-63. doi: 10.12691/ajeee-1-3-5.

\section{Introduction}

High power semiconductor laser diodes have occupied the minds of researchers in the last decade due to the emerging widespread usage in the fields of medicine, industry and in telecommunications [1,2,3].

The effectiveness of Barlase has already been demonstrated using hypothetical laser bars and published elsewhere [4]. In this paper, Barlase is being used in its upgraded form by introducing a global thermal solver to emulate the degradation processes of a real laser bar with 16 emitters. This is being done by comparing experimental results with the emulation results in order to establish a correlation between them.

This paper gives a further credence to the by-emitter degradation analysis technique developed over recent years Xia et al. [5,6], Bull et al. [7,8] and Bream et al. [9]. This tool is also an addition to the by-emitter analysis technique where the effects of certain factors that affect the degradation of laser emitters/bars can be investigated. The objective of this study is to establish a correlation between experimental and emulated results using an upgraded version of Barlase to demonstrate the versatility of the emulation tool Barlase.

\section{Materials and Methods}

The bar emulation model was enhanced by including a global thermal solver (GTS) to model the thermal crosstalk between emitters. This was done by alternating between the electrical-optical solver applied for each of the emitters and the heat diffusion equation applied to the entire bar, including the substrate and heatsink. The GTS solves the heat flow equation:

$$
\operatorname{div}(-\kappa(T) \operatorname{grad} T)=H
$$

where $\kappa$ is the thermal conductivity, grad $T$ is the temperature gradient and $H$ is the heat source distribution. The heat flow equation is also solved using Newton's method Lim et al. [10]. Figure 1 demonstrates the flow diagram of the bar emulation tool showing the electroopto-thermal communication between the solvers. The analysis is initiated at the rear facet and the optical field is propagated a distance to the next electrical slice, yielding a new photon density distribution. The new photon distribution is used to obtain the new carrier and potential distributions in the cavity. These new carrier profiles are used to update the lateral gain and refractive index 
distribution for the optical propagation to the next electrical slice Sujecki et al. [11].

The electrical solver also calculates the heat sources from the individual emitter simulations and supplies them to the global thermal solver. The heat sources include the Joule heating, nonradiative recombination heating, free carrier absorption heating and capture heating. The thermal distribution is updated by the GTS at each electrothermal slice. The GTS calculates the temperature distribution for the entire 2D cross-section of the bar by solving the heat flow equation (1). The global thermal distribution will be saved until the next instance of the electrical solver is called at that position. The thermal distribution is used to update all the variables in the electrical solver, which depend on temperature. These include the bandgap energy, the carrier mobilities, gain, recombination rates and carrier density.

The thermal conductivities of the different materials in the bar are supplied to the GTS. These materials include the epitaxial structure, the heatsink, the metal contacts, and the polymer used to fill the etched trenches of the ridge-waveguide. Figure 2 shows the top view and the transverse cross section of the $975 \mathrm{~nm}$ tapered laser bar.

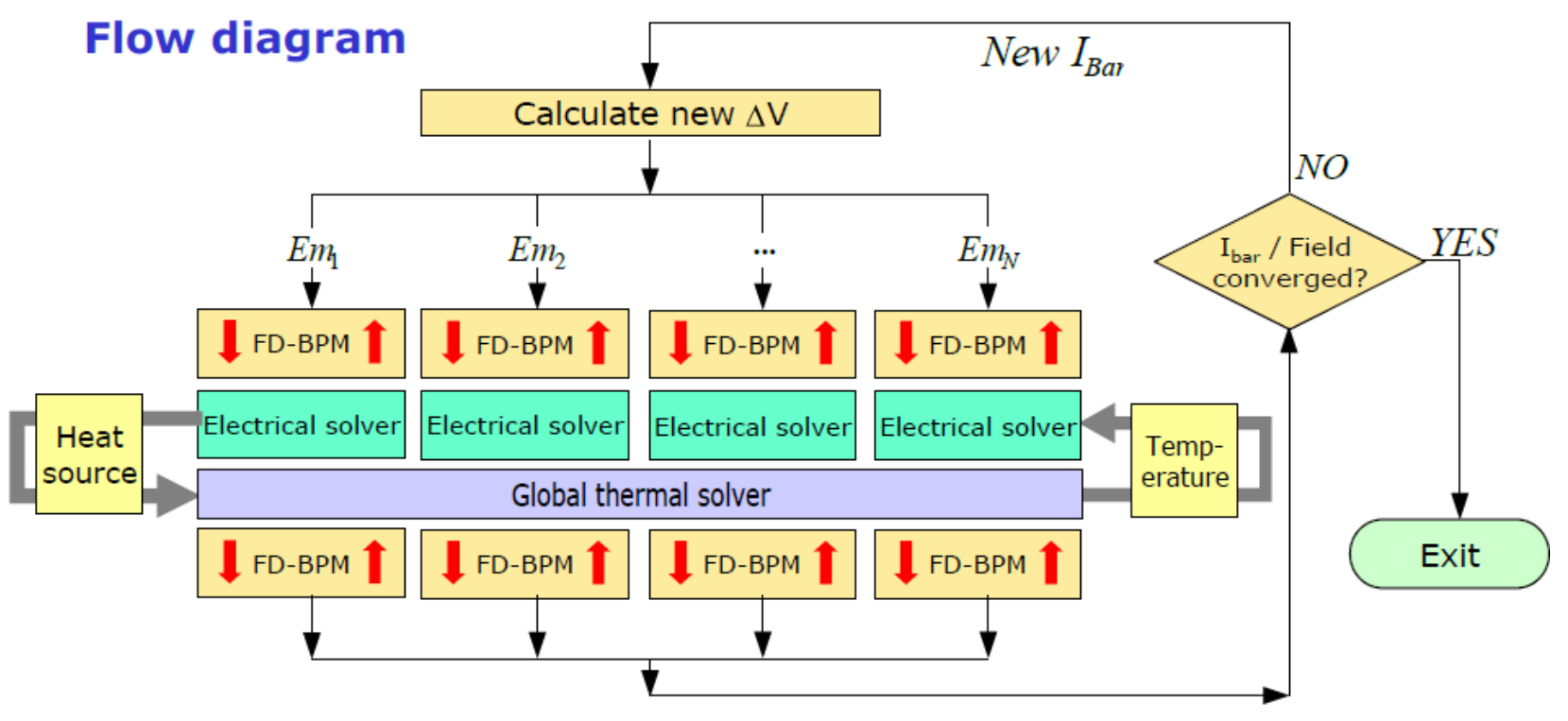

Figure 1. Flow diagram of the global thermal solver showing the electro-optical communication in the bar emulation tool

$\leftrightarrow 50 \mu \mathrm{m}$

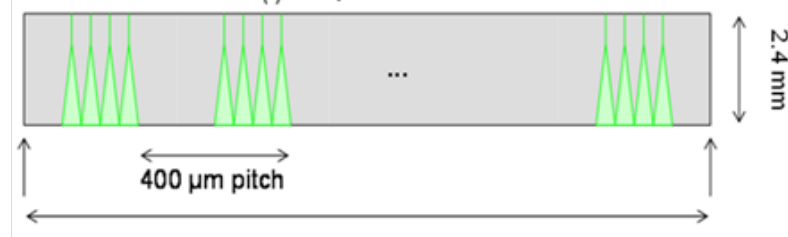

16 groups of $4=64$ emitters $=6.4 \mathrm{~mm}$

(a)

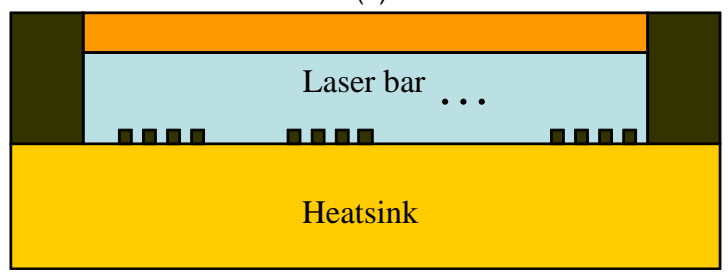

(b)

Figure 2. (a) Top view of bar and (b) transverse cross section of the bar

The Fox-Li iterations are performed for each emitter until convergence is achieved in the output power. The operations of the GTS can be summarised as follows:

- For each emitter group, electro-optical simulations are performed on 1 emitter.

- The heat source distribution is calculated and supplied to the global thermal solver. Identical heat sources are used for each emitter in the group.

- The temperature distribution for the entire bar is solved and the average temperature for an emitter in the group is calculated and fed back to Speclase and

- The global thermal solver includes all 64 emitters.

\section{Results and Discussion}

The GTS has definitely brought an improvement in the bar emulation model judging from the output current/power profiles in Figure 3. Clearly defined frown shaped profiles are exhibited by the output current and power. However, the maximum variation in power is only about $0.75 \%$ which is very small compared to experimental results $\sim 50-60 \%$.

Higher currents were drawn and consequently more power emitted towards the centre of the bar compared to the edge [12], Amuzuvi et al. [13]. The temperature at the front facet is higher and has a stronger curvature than at the back facet, which is expected (Figure 4). This is because the front facet is the part of the laser array which draws more current and is more exposed to local heating of the laser mirrors [14]. The lifetime of high-quality GaAs-based lasers is often no longer limited by failures in the bulk semiconductor, but rather by degradation processes in the susceptible mirror facets [15].

Light absorption (especially at the front facet), and the recombination current density increase with temperature, due to local thermal shrinkage of the bandgap energy. The bandgap shrinkage in turn, is caused by heat from the recombination current [16]. Thermal runaway in semiconductor lasers (which is as a result of facet heating) leads to catastrophic damage and occurs predominantly at the front facet. It is generally believed that thermal runaway is a positive feedback process initiated by heat 
created from intense nonradiative recombination [17]. This feedback, which occurs at certain output powers, leads to a rapid temperature increase that result in local

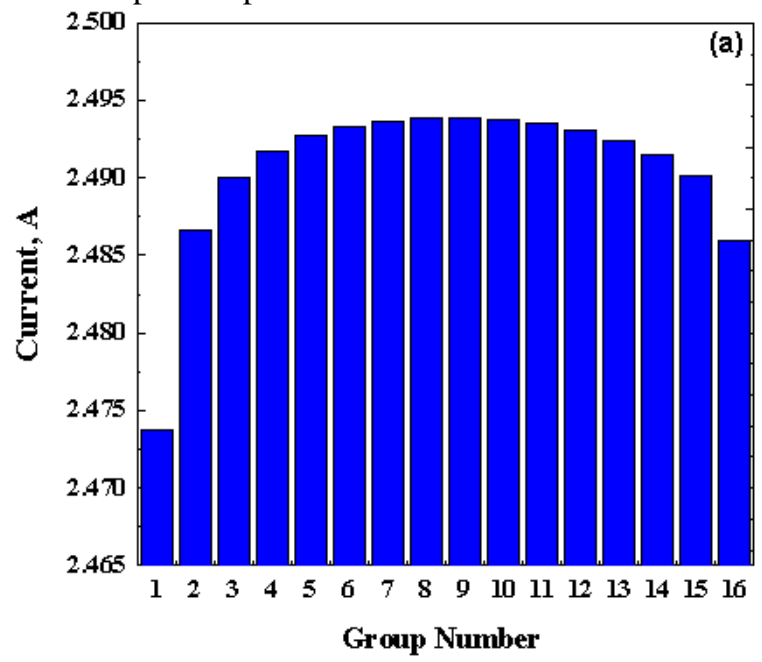

melting of the active material and catastrophic damage of the laser front facet.

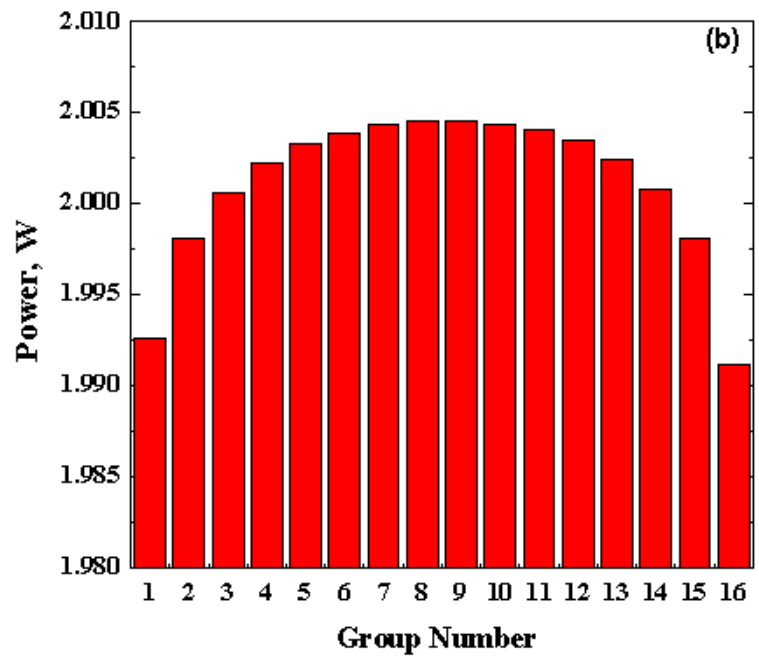

Figure 3. (a) Current and (b) power versus emitter group numbers
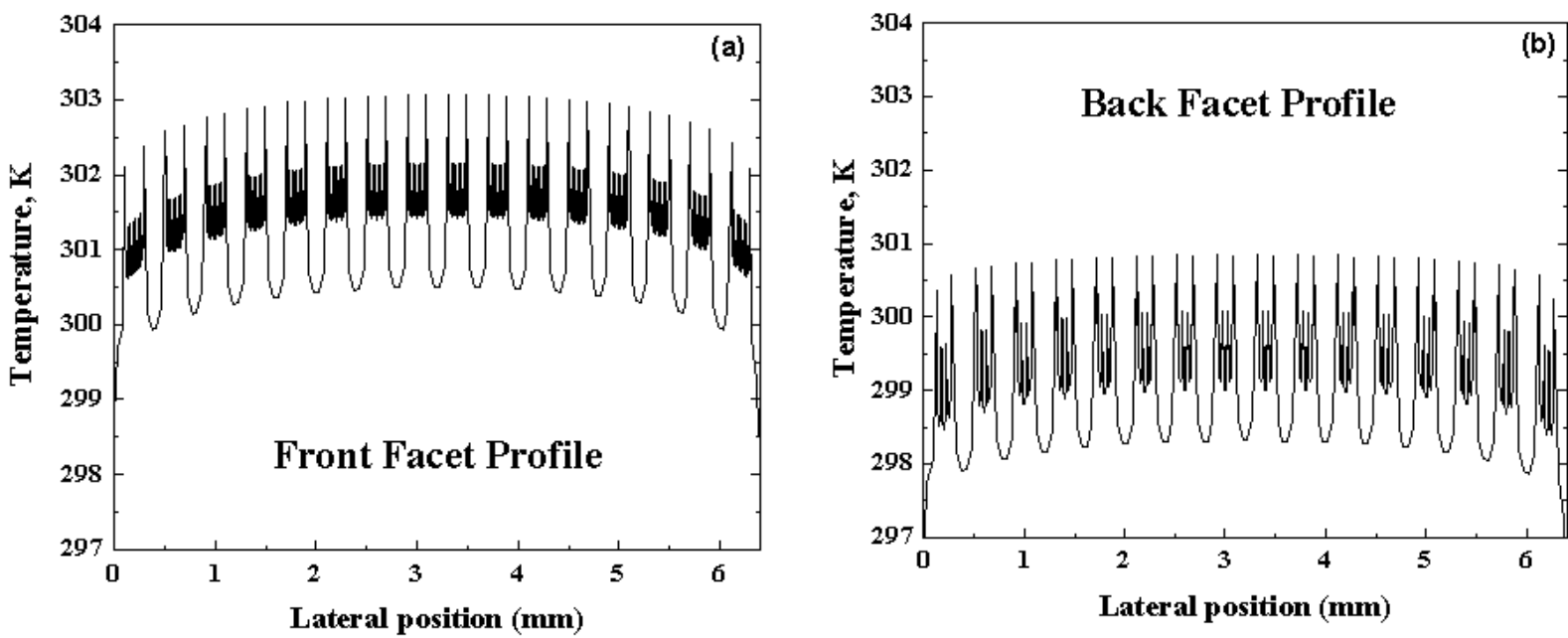

Figure 4. (a) Front facet temperature profile of the bar and (b) back facet temperature profile of the bar

Various experiments, among them facet temperature measurements for ultra high-power operation have provided evidence that in arrays, the front facets are significantly affected by device operation mainly due to front facet heating Tomm et al. [18]. Therefore, it is not surprising that the front facet as shown in Figure 4a has a higher temperature than the back facet in Figure $4 \mathrm{~b}$.

The variations though, in the current and power as indicated in Figure 3 is less pronounced than what pertains in the experimental results. The reasons for this could be investigated in further work on this research.

\section{Conclusion}

Emulation has been attempted for a real bar, whose performance and degradation has been characterised in detail using by-emitter degradation analysis. Although the simulated output power profile did not correspond well with the experimental power profile, there was good agreement between the total output power of the bar. Better correlation was observed between the experimental and the simulated temperature profiles. This was expected since the experimental temperature was set as input for the heatsink temperature profile. The agreement therefore must not be over-emphasised. The bar emulation model was enhanced by including a global thermal solver to model effectively the thermal crosstalk between emitters. Emulations using this model showed a clearly defined frown shaped profile in the output current and power profiles but the change was minimal. As the emulation of laser bar degradation has not been attempted before, this work is still at a very early stage. Further work is needed to achieve better agreement in the output current/power profiles to better the model.

\section{Acknowledgment}

CKA thanks the University of Mines and Technology, Tarkwa, Ghana and the GetFund for their support.

\section{References}

[1] Schulz, W. and R. Poprawe, 2000. "Manufacturing with novel high-power diode lasers," IEEE Journal of Selected Topics in Quantum Electronics, vol. 6, pp. 696-705. 
[2] Gannot, I and R. W. Waynant, 2001. "Introduction to the issue on lasers in medicine and biology”, IEEE Journal of Selected Topics in Quantum Electronics, vol. 7, pp. 873-873.

[3] Rochat, E., K. Haroud, and R. Dandliker, 1999. "High-power Nddoped fiber amplifier for coherent intersatellite links", IEEE Journal of Quantum Electronics, vol. 35, pp. 1419-1423.

[4] Amuzuvi, C. K. and J. C. Attachie, 2013. "Describing a Laser Diode Emulation Tool Using Single Emitter Simulation Results”. Research Journal of Applied Sciences, Engineering and Technology, 5(04): 1358-1361.

[5] Xia, R., E. C. Larkins, I. Harrison, S. R. A. Dods, A. V. Andrianov, J. Morgan and J. P. Landesman, 2002. "Mounting-induced strain threshold for the degradation of high-power AlGaAs laser bars," IEEE Photon. Technol. Lett., 14, 893.

[6] Tomm J. W., A. Gerhardt, T. Elsaesser, D. Lorenzen, and P. Hennig, 2002. "Simultaneous quantification of strain and defects in high-power diode laser devices”, Applied Physics Letters, vol. 81, pp. 3269-3271.

[7] Bull S., J. W. Tomm, M. Oudart, J. Nagle, C. Scholz, K. Boucke, I. Harrison, and E. C. Larkins, 2005. "By-emitter degradation analysis of high-power laser bars”, Journal of Applied Physics, vol. 98, p. 063101.

[8] Lim, J. J., T. M. Benson and E. C. Larkins, 2005 "Design of wideemitter single-mode laser diodes,” IEEE J. Quantum Electron., 41, 506.

[9] Bream, P. J., J. J. Lim, S. Bull, A. V. Andrianov, S. Sujecki and E. C. Larkins, 2006. "The impact of nonequilibrium gain in a spectral laser diode model,” Opt. Quantum Electron., 38, 1019.

[10] Lim J. J., S. Sujecki, L. Lang, Z. C. Zhang, D. Paboeuf, G. Pauliat, G. Lucas-Leclin, P. Georges, R. C. I. MacKenzie, P. Bream, S. Bull, K. H. Hasler, B. Sumpf, H. Wenzel, G. Erbert, B. Thestrup, P. M. Petersen, N. Michel, M. Krakowski, and E. C. Larkins, "Design and Simulation of Next-Generation High-Power, High-
Brightness Laser Diodes,” IEEE Journal of Selected Topics in Quantum Electronics, vol. 15, pp. 993-1008, May-Jun 2009.

[11] Sujecki S., L. Borruel, J. Wykes, P. Moreno, B. Sumpf, P. Sewell, H. Wenzel, T. A. Benson, G. Erbert, I. Esquivias, and E. C. Larkins, "Nonlinear properties of tapered laser cavities," IEEE Journal of Selected Topics in Quantum Electronics, vol. 9, pp. 823-834, May-Jun 2003.

[12] Amuzuvi C. K., S. Bull, J. J. Lim, S. Sujecki, and E. C. Larkins, "Numerical emulation of the degradation of $975 \mathrm{~nm}$ high power tapered laser bars," High Power Diode Lasers and Systems Conference, Coventry (UK) 2009. HPD 2009, 2009, pp. 1-2.

[13] Amuzuvi C. K., S. Bull, J. W. Tomm, J. Nagle, E. C. Larkins, B. Sumpf, G. Erbert, N. Michel, and M. Krakowski, “The impact of temperature and packaging-induced strain on current competition and emitter power in laser bars,” Applied Physics Letter, June 2011.

[14] Chen G. and C. L. Tien, "Facet heating of quantum-well lasers," Journal of Applied Physics, vol. 74, pp. 2167-2174, Aug 1993.

[15] Moser A., "Thermodynamics of facet damage in cleaved AlGaAs lasers,” Applied Physics Letters, vol. 59, pp. 522-524, 1991.

[16] Schatz R. and C. G. Bethea, "Steady-state model for facet heating leading to thermal runaway in semiconductor-lasers," Journal of Applied Physics, vol. 76, pp. 2509-2521, Aug 1994.

[17] Henry C. H., P. M. Petroff, R. A. Logan, and F. R. Merritt, "Catastrophic damage of AlxGa1-xAs double-heterostructure laser material,” Journal of Applied Physics, vol. 50, pp. 3721-3732, 1979.

[18] Tomm J. W., E. Thamm, A. Barwolff, T. Elsaesser, J. Luft, M. Baeumler, S. Mueller, W. Jantz, I. Rechenberg, and G. Erbert, "Facet degradation of high-power diode laser arrays," Applied Physics a-Materials Science \& Processing, vol. 70, pp. 377-381, 2000. 